\title{
Establishment of a new animal model of allergic rhinitis with biphasic sneezing by intranasal sensitization with Staphylococcal enterotoxin B
}

\author{
RONG SUN ${ }^{1}$, XINYE TANG ${ }^{2}$, HONGBING YAO $^{2}$, SULING HONG $^{3}$, \\ YANG YANG $^{2}$, WEI KOU ${ }^{2}$ and PING WEI ${ }^{2}$ \\ ${ }^{1}$ Department of Physical Examination, The First Affiliated Hospital of Chongqing Medical University; \\ ${ }^{2}$ Department of Otolaryngology, Children's Hospital of Chongqing Medical University, \\ Pediatric Research Institute of Chongqing Medical University, National Key Disciplinary Areas of Pediatrics, \\ Ministry of Education; ${ }^{3}$ Department of Otolaryngology-Head and Neck Surgery, \\ The First Affiliated Hospital of Chongqing Medical University, Chongqing, P.R. China
}

Received July 14, 2014; Accepted February 16, 2015

DOI: $10.3892 / \mathrm{etm} .2015 .2506$

\begin{abstract}
Allergic rhinitis (AR) is a global health problem. The effectiveness of currently available medications is limited and therefore investigation for more effective drugs is essential. The aim of the present study was to establish a model of AR in guinea pigs that can be utilized for the further investigation of new drugs. Guinea pigs were intranasally sensitized with $1 \mu \mathrm{g}$ Staphylococcal enterotoxin B (SEB) dissolved in $40 \mu \mathrm{l}$ saline once daily for 14 days. One week after the last sensitization, the same treatment was applied intranasally once every four days for a total of 30 times. In the treatment group, terfenadine was administered orally $70 \mathrm{~min}$ before the 4th, 14th and 24th challenge. Sneezing and nasal scratching were evaluated following each of the 30 challenges. The quantity of antigen-specific antibodies in the serum was measured. Between the 19th and 30 th challenges, the guinea pigs in the model group produced significant biphasic elevations in sneezing number, with peaks 10 min- $2 \mathrm{~h}$ and $4-8 \mathrm{~h}$ after the SEB challenges. In addition, the guinea pigs produced significantly more sneezing in the first peak during the 19th to 30th challenges than during the first to 18th challenges $(\mathrm{P}<0.01)$. Terfenadine significantly inhibited the early- and late-phase sneezing at all challenge times. The serum levels of SEB-specific immunoglobulin (Ig) $\mathrm{E}$ and $\mathrm{IgG}_{1}$ were higher in the model group in comparison with those in
\end{abstract}

Correspondence to: Mr. Xinye Tang, Department of Otolaryngology, Children's Hospital of Chongqing Medical University, Pediatric Research Institute of Chongqing Medical University, National Key Disciplinary Areas of Pediatrics, Ministry of Education, 136 Zhongshan 2 Road, Chongqing 400014, P.R. China

E-mail: ent2002@163.com

Key words: allergic rhinitis, Staphylococcal enterotoxin B, immunoglobulin $\mathrm{E}$, immunoglobulin $\mathrm{G}_{1}$, guinea pig, eosinophil, sneeze, histamine the control group $(\mathrm{P}<0.01)$. This experiment demonstrated that SEB can induce typical AR with biphasic sneezing in guinea pigs. Histamine may play an important role in the early- and the late-phase sneezing in the model of AR. This model can be potentially used for the investigation of new drugs.

\section{Introduction}

Allergic rhinitis (AR) is an atopic disease characterized by the clinical symptoms of sneezing, itching, rhinorrhea and nasal blockage. Antigen provocation tests have indicated that a biphasic reaction occurs in the respiratory tract. The early phase of the immediate reaction starts minutes after allergen provocation. The nasal symptoms reappear 5-10 h after allergen provocation, which is known as the late-phase reaction (LPR) (1-4). In previous studies, the majority of patients suffering from AR showed the above symptoms immediately, while $>50 \%$ of them developed an LPR following antigen challenge (1-4). It has been reported that sneezing and rhinorrhea are the strongest symptoms during the allergic LPR (5-7), and studies have found that $>90 \%$ of patients showed nasal blockage in the LPR $(8,9)$. There are numerous animal models that can be used to study the LPR of AR $(10,11)$; however, these only show nasal blockage in the LPR. To date, no animal models have been reported to show AR with early- and late-phase sneezing.

Staphylococcal enterotoxin B (SEB), produced by Staphylococcus aureus, is an exotoxin that may act as allergen or superantigen to induce allergic disease. We have previously described an AR model developed by repeated intranasal instillation with SEB in guinea pigs (12). The model, which exhibited the typical symptoms of AR, showed sneezing and nasal scratching, thereby demonstrating that SEB has the potential to act as a conventional allergen to induce AR. The nasal cavity and skin of humans are the most common sites for Staphylococcus aureus colonization $(13,14)$. In excess of $50 \%$ of pathogenic isolates of Staphylococcus aureus produce one or more superantigen exotoxins (15). This increases the risk that the atopic individual suffers from allergic disease. In the present 
study, the aim was to describe a novel method for establishing experimental AR with early and late sneezing with SEB in guinea pigs.

\section{Materials and methods}

Animals and materials. Male, eight-week-old, healthy Hartley guinea pigs (250-300 g) were purchased from the Laboratory Animal Center of Chongqing Medical University (Chongqing, China). The animals were housed in an air-conditioned room in which allergen-free conditions were maintained. The animals were fed according to the Institutional Guidelines for the Care and Use of Laboratory Animals. All experimental procedures on animals were approved by the Ethics Committee of Chongqing Medical University. SEB and terfenadine were purchased from Sigma (St. Louis, MO, USA).

Sensitization and challenge. As shown in Fig. 1, guinea pigs ( $\mathrm{n}=10$ per group) were intranasally sensitized with $1 \mu \mathrm{g} \mathrm{SEB}$ dissolved in $40 \mu \mathrm{l}$ saline in the absence of adjuvant once every day for 14 days. Prior to each sensitization, the upper airway mucosal surface was anesthetized by intranasal instillation of $4 \%$ lidocaine hydrochloride solution (China Otsuka Pharmaceutical Co., Ltd., Tianjin, China) to prevent the rapid elimination of antigen by ciliary movement (8). One week after the final sensitization, the same treatment was applied intranasally once every four days for a total of 30 times. For the control group, the animals were treated with the same volume of saline instead of SEB. In addition, the effects of histamine in AR were evaluated. In the treatment group, terfenadine $(20 \mathrm{mg} / \mathrm{kg})$ was administered orally $70 \mathrm{~min}$ before the 4th, 14th and 24th challenge. Terfenadine is an $\mathrm{H} 1$ receptor antagonist. It has been reported that terfenadine at the above doses significantly inhibits allergic inflammation of the nasal cavity induced by histamine in guinea pigs (16).

Observation of symptoms. The number of sneezes in the intervals $0-10 \mathrm{~min}, 10 \mathrm{~min}-2 \mathrm{~h}, 2-4 \mathrm{~h}, 4-6 \mathrm{~h}, 6-8 \mathrm{~h}$ and $8-10 \mathrm{~h}$ after the SEB intranasal challenge was counted. The nasal scratching number was measured simultaneously. The symptom observation was performed manually in each animal.

Serum immunoglobulin (Ig) levels. Ten hours after the final challenge, the guinea pigs were anesthetized intramuscularly with urethane $(1.35 \mathrm{~g} / \mathrm{kg}$; Sinopharm Chemical Reagent Co., Ltd., Shanghai, China). The animals were then sacrificed by cardiac puncture and the serum was collected. Serum SEB-specific $\mathrm{IgG}_{1}$ levels were measured with indirect ELISA, as previously described (12). In brief, the plates were coated (100 $\mu \mathrm{l} /$ well) with $0.1 \mu \mathrm{g} / \mathrm{ml} \mathrm{SEB}$ in $0.05 \mathrm{M} \mathrm{NaHCO}_{3}$ (pH 9.6) at $4^{\circ} \mathrm{C}$ overnight. The plates were washed with phosphate-buffered saline-Tween 20 and blocked $(200 \mu \mathrm{l} /$ well $)$ with $2 \%$ bovine serum albumin (BSA) for $2 \mathrm{~h}$ at $20^{\circ} \mathrm{C}$ and re-washed. Sera diluted $1: 100$ in $0.1 \%$ BSA were incubated (100 $\mu \mathrm{l} /$ well) at $4^{\circ} \mathrm{C}$ overnight. Following a washing step, 1:500 diluted horseradish peroxidase (HRP)-goat anti-guinea pig $\operatorname{IgG}_{1}$ monoclonal antibody (mAb) (\#AHP861P; AbD SeroTec, Oxford, UK) in $0.1 \%$ BSA was incubated $(100 \mu \mathrm{l} /$ well $)$ at $20^{\circ} \mathrm{C}$ for $2 \mathrm{~h}$, prior to a second washing step. Tetramethylbenzidine chromogenic reagent (100 $\mu$; Boster Biological Technology, Ltd., Wuhan,
China) was incubated at $20^{\circ} \mathrm{C}$ for $10 \mathrm{~min}$ and Stop Solution (100 $\mu \mathrm{l} /$ well; Boster Biological Technology, Ltd.) was added. Titers for SEB-specific $\operatorname{IgG}_{1}$ were estimated as mean optical density (OD) at $450 \mathrm{~nm}$. SEB-specific $\mathrm{IgG}_{2}$ and $\mathrm{IgE}$ levels were measured by the same aforementioned method. Specific $\mathrm{IgG}_{2}$ was detected with HRP-goat anti-guinea pig $\operatorname{IgG}_{2} \mathrm{mAb}_{(1: 500 \text {; }}$ \#AHP862P; AbD SeroTec), and specific IgE was detected with HRP-goat anti-mouse IgE mAb (1:500; \#1110-05; Southern Biotech, Inc., Birmingham, AL, USA).

Histological examination. Samples of nose, trachea, bronchi and lungs were collected, fixed in formalin and embedded in paraffin. Transverse sections were cut and stained with hematoxylin and eosin. Initially, eosinophil infiltration in the nasal mucosa was observed microscopically in a high-power field. A total of 200 leukocytes were then counted microscopically (magnification, 10x100) in total. The percentage of eosinophils among the total leukocytes was counted.

Statistical analysis. All results with a normal distribution are expressed as the mean \pm standard deviation. Comparisons of means between different groups were performed with a Student's t-test (two groups). Data presenting in a non-normal distribution are expressed as median (Q). Statistical analyses were performed with the Wilcoxon test. $\mathrm{P}<0.05$ was considered to indicate a statistically significant difference.

\section{Results}

Changes in the sneezing and nasal scratching number. Between the first and 12th challenges, the guinea pigs in the model group produced a high sneezing number within $10 \mathrm{~min}$ after each challenge. From the 10th min, little or no sneezing was observed at any challenge time. The total number of sneezes increased with increasing challenge number (Fig. 2A-D). Between the 13th and 18th challenges, no notable increase in sneezing number was observed among the SEB-sensitized guinea pigs compared with the sneezing number of the earlier challenges (Fig. 2E). Between the 19th and 30th challenges, the guinea pigs in the model group experienced a significant biphasic increase in the number of sneezes, reaching multiple peaks at $10 \mathrm{~min}-2 \mathrm{~h}$ and 4-8 $\mathrm{h}$ after SEB challenge. The first peak in sneezing number shifted from the first $10 \mathrm{~min}$ to the period of $10 \mathrm{~min}-2 \mathrm{~h}$ after SEB challenge. In addition, the first sneezing number peak was significantly higher between the 19th and 30th challenges than that between the first and 18th challenges $(\mathrm{P}<0.01)$ (Fig. 2F-H). Furthermore, terfenadine significantly reduced the early and the late elevations in sneezing number at the 24th challenge $(\mathrm{P}<0.01)$ (Fig. 3A-C). The guinea pigs in the control group produced significantly less sneezing than those in the model group $(\mathrm{P}<0.01)$. The changes in nasal scratching number were similar to the changes in sneezing number (data not shown).

Ig production in the serum. In the model group, the guinea pigs produced increased levels of SEB-specific $\operatorname{IgE}$ and $\mathrm{IgG}_{1}$. The mean ODs at $450 \mathrm{~nm}$ were $0.23 \pm 0.06$ and $4.21 \pm 0.28$, respectively. By contrast, levels of SEB-specific $\operatorname{IgE}$ and $\operatorname{IgG}_{1}$ in the control group could not be detected. Compared with the control group, the increases observed in the model group were statistically significant $(\mathrm{P}<0.01$, Wilcoxon test) (Fig. $4 \mathrm{~A}$ and $\mathrm{B})$. In the 


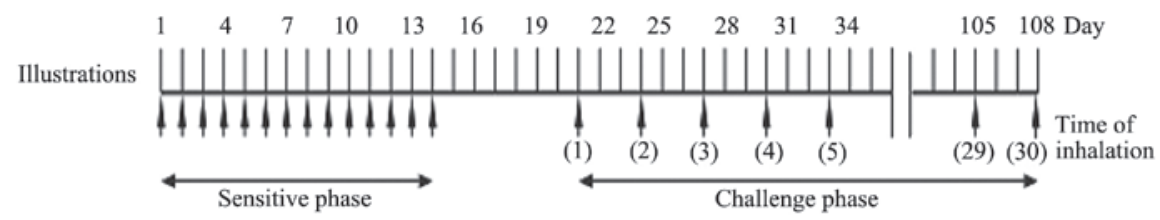

Figure 1. Experimental outline of the sensitization and challenge periods.

A

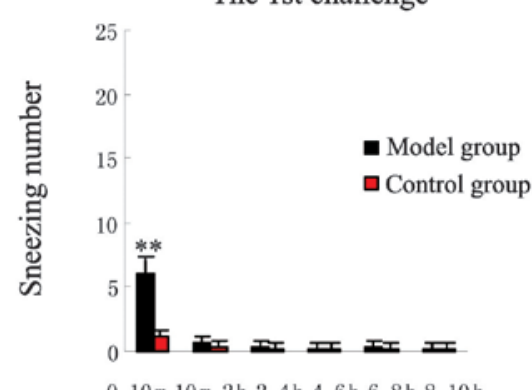

C

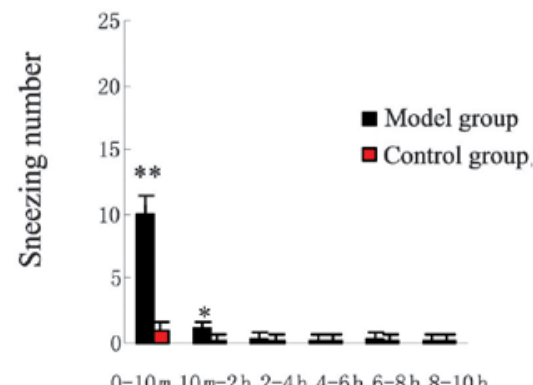

$\mathbf{E}$

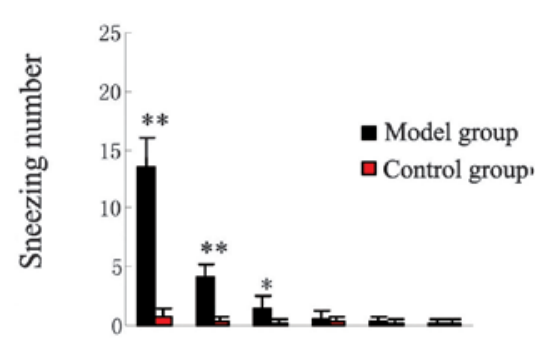

0-10m 10m-2h 2-4h 4-6h 6-8h 8-10h
B The 3rd challenge

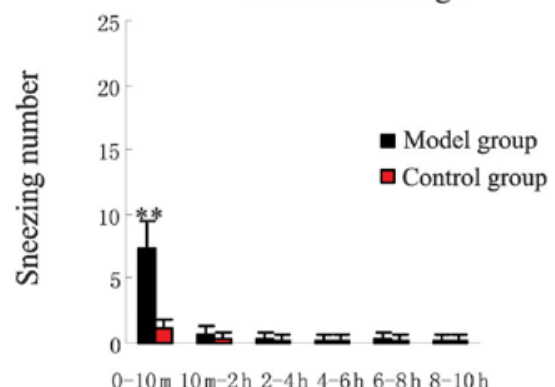

D The 12th challenge

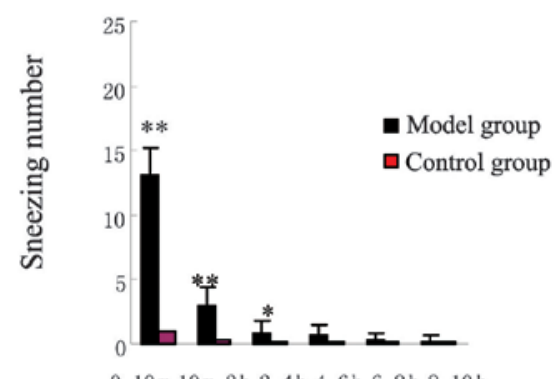

0-10m 10 m-2h 2-4h 4-6h 6-8h 8-10h

F

The 19th challenge

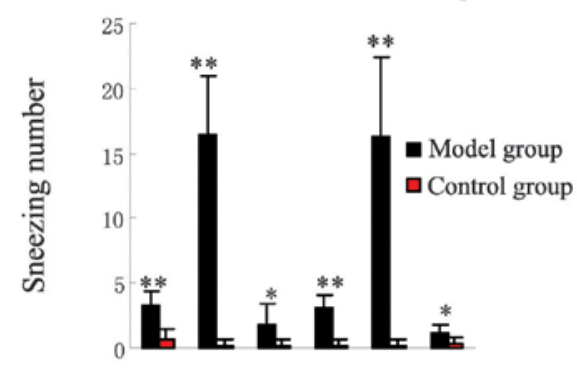

0-10m $10 \mathrm{~m}-2$ h $2-4$ h $4-6$ h $6-8$ h $8-10$ h
G

The 26th challenge

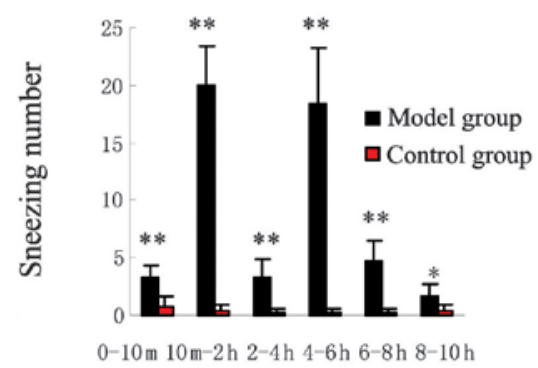

H The 30th challenge

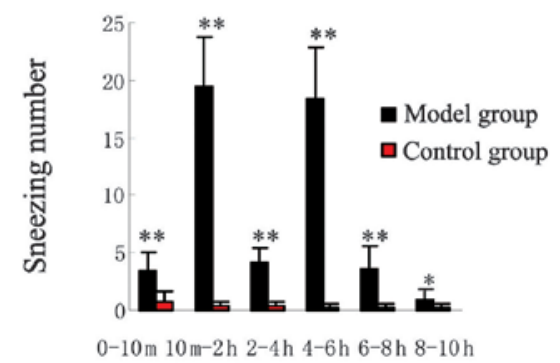

Figure 2. Changes in sneezing number following intranasal instillation with SEB and saline at the (A) 1st, (B) 3rd, (C) 5th, (D) 12th, (E) 16th, (F) 19th, (G) 26th and $(\mathrm{H}) 30$ th challenges. Data are presented as the mean \pm standard deviation from 10 guinea pigs. Between the 1st and 18th challenges, the guinea pigs with SEB challenge produced a significantly higher sneezing number only within the first $10 \mathrm{~min}$ after each challenge. Between the 19th and 30th challenges, the guinea pigs with SEB challenge produced significant biphasic elevations in sneezing number, with peaks appearing $10 \mathrm{~min}-2 \mathrm{~h}$ and $4-8 \mathrm{~h}$ after the SEB challenge. ${ }^{*} \mathrm{P}<0.05$ and $^{* *} \mathrm{P}<0.01$ compared with the control group. SEB, Staphylococcal enterotoxin B. 
A

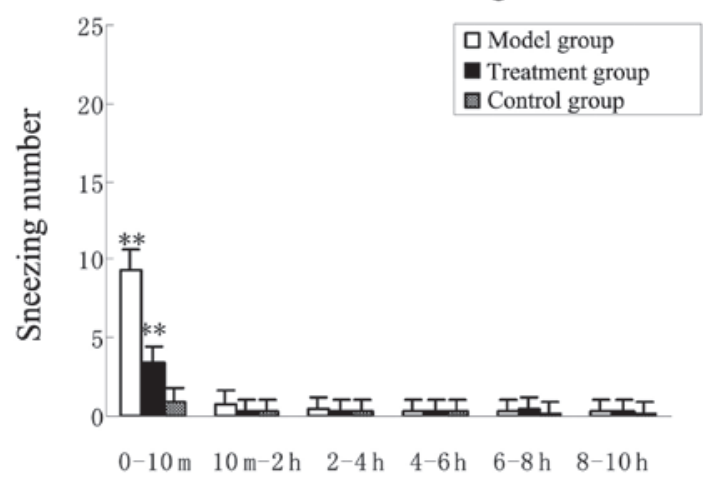

B

The 14th challenge

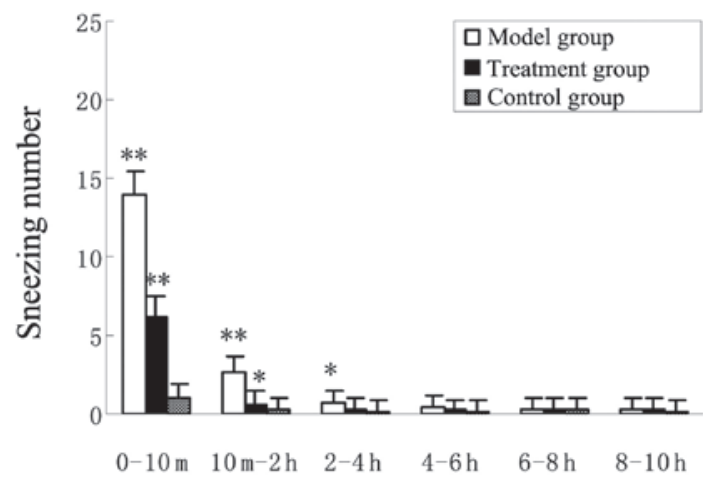

C

The 24th challenge

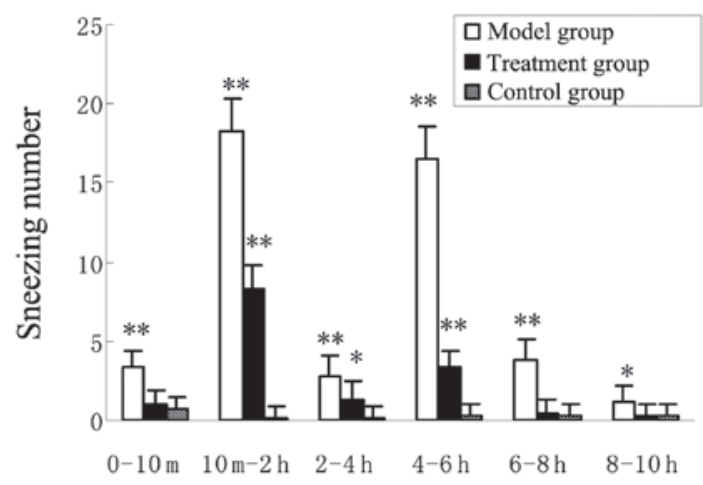

Figure 3. Effects of terfenadine on sneezing number at the (A) 4th, (B) 14th and (C) 24th challenges. Data are presented as the mean \pm standard deviation from 10 guinea pigs. At the 4th and 14th challenges, the guinea pigs with SEB challenge produced a significantly higher sneezing number only within the first 10 min after challenge. Biphasic elevation in the sneezing number was observed at $10 \mathrm{~min}-2 \mathrm{~h}$ and $4-6 \mathrm{~h}$ after the 24th SEB challenge. Terfenadine significantly reduced the early and late elevation in sneezing number. ${ }^{*} \mathrm{P}<0.05$ and ${ }^{* *} \mathrm{P}<0.01$ compared with the control group. SEB, Staphylococcal enterotoxin $\mathrm{B}$.

two groups, the guinea pigs did not produce detectable amounts of SEB-specific $\operatorname{IgG}_{2}$ (Fig. 4C).

Eosinophils in the nasal mucosa. In the model group, the guinea pigs showed apparent eosinophil infiltration in the nasal mucosa. The eosinophils infiltrated not only the lamina propria but also the epithelial layer (Fig. 5A); however, eosinophil infiltration was not observed in the guinea pigs challenged with saline (Fig. 5B). In the model group, eosinophils accounted for
A

SEB-specific IgE

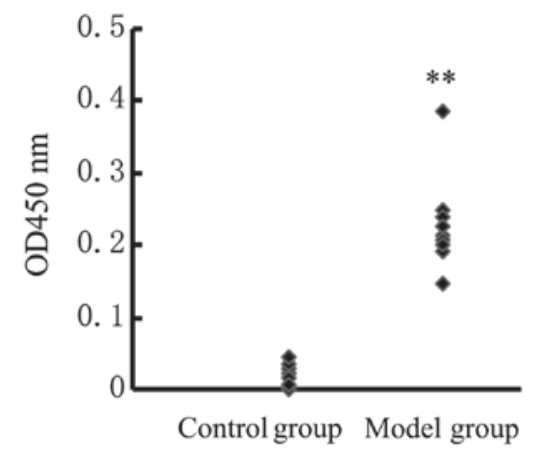

B

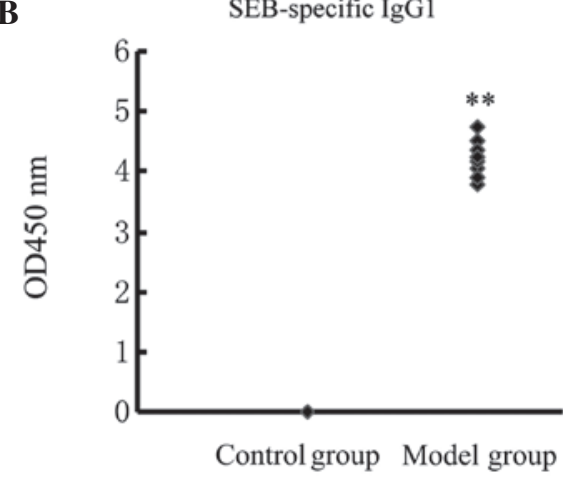

C

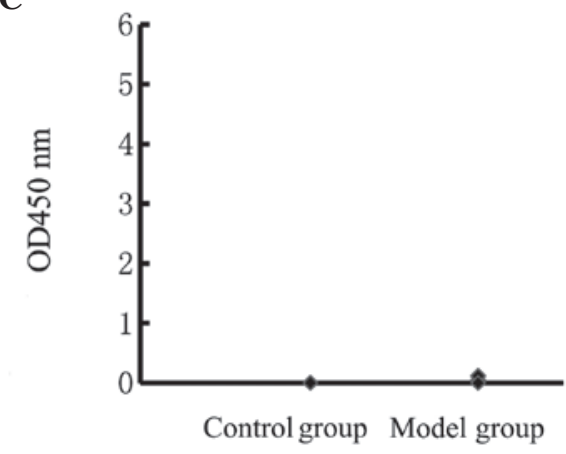

Figure 4. SEB-specific (A) $\operatorname{IgE}$,(B) $\operatorname{IgG}_{1}$ and (C) $\mathrm{IgG}_{2}$ levels in the serum in SEB- and saline-challenged guinea pigs. In the model group, the levels of SEB-specific $\operatorname{IgE}$ and $\operatorname{IgG}_{1}$ were $0.23 \pm 0.06$ and $4.21 \pm 0.28$, respectively. By contrast, levels of SEB-specific $\operatorname{IgE}$ and $\mathrm{IgG}_{1}$ could not be detected in the control group. Compared with the control group, the increases observed in the model group were statistically significant $(\mathrm{P}<0.01)$. ${ }^{*} \mathrm{P}<0.05$ and ${ }^{* *} \mathrm{P}<0.01$ compared with the control group. SEB, Staphylococcal enterotoxin B; Ig, immunoglobulin; OD, optical density.

$49.5 \%$ (median quartile, 0.107 ) of the total leukocytes, compared with only $3.1 \%$ (median quartile, 0.015 ) in the control group. Compared with the control group, this increase was statistically significant $(\mathrm{P}<0.01$, Wilcoxon test $)$. Furthermore, vasodilatation and edema were present in the submucosal areas among the SEB-challenged guinea pigs. The trachea, bronchi and lungs in the two groups exhibited normal tissue without significant pathological changes.

\section{Discussion}

AR is a global health problem. The successful study of the pathogenesis and pathophysiology is dependent upon the 


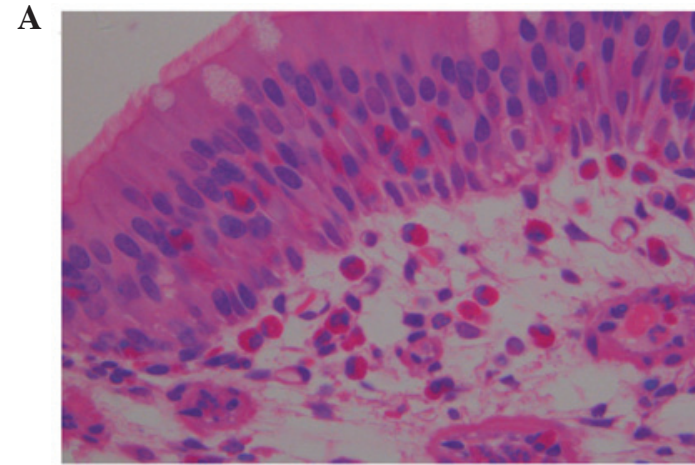

B

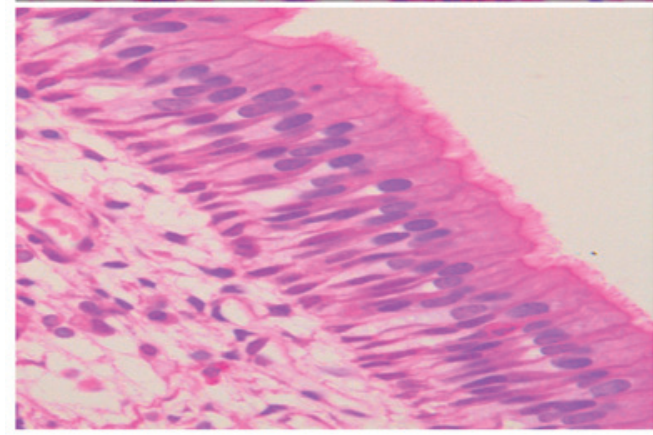

Figure 5. Histological features of the nasal mucosa in (A) SEB- and (B) saline-challenged guinea pigs (hematoxylin and eosin staining; magnification, 10x100). Compared with the guinea pigs challenged with saline (B), guinea pigs challenged with SEB (A) exhibited evident eosinophil infiltration, vasodilatation and edema in the nasal mucosa. SEB, Staphylococcal enterotoxin B.

establishment of a successful animal model of AR; however no unified standards can be used at present. According to the literature, several sensitization methods, such as intraperitoneal and intradermal injections and inhalation, have been used to study AR (17). Animal models induced by intraperitoneal and intradermal injections of allergens vary greatly from the natural processes of AR. Furthermore, aerosol inhalation is likely to cause sensitization of the lower respiratory tract, which can easily induce asthma (18). In the present study, the intranasal instillation method was used for the sensitization and challenge processes; the aim of this was to ensure that the allergic inflammation was confined to the nasal cavity, consistent with the natural process of AR. Previous studies concerning animal models of AR have reported that the time required to build an animal model varies greatly, from one week to several months $(10,11,19-25)$. In general, animal models that can be established in a short time can only reflect the early-phase symptoms; models facilitating the observation of the LPR can take a long time to build. Nabe et al (10) have established an animal model of AR with the biphasic nasal blockage by Japanese cedar pollen. In their model, biphasic nasal blockage was evoked 6-7 weeks after the first sensitization. In the present study, biphasic sneezing was exhibited by the guinea pigs following the 19th nasal challenge (model day 75).

To date, investigations of the LPR have focused on nasal blockage in the animal models of AR (26-28). These reports describe that nasal blockage is a predominant symptom in the LPR. Sneezing is a major symptom in the early-phase reaction; however, in the present study it was demonstrated that SEB could act as a type of allergen, by repeatedly stimulating nasal tissue, and induce typical symptoms of AR with a biphasic response. It was found in this study that frequent sneezing was a significant feature of the LPR, and exhibited a biphasic response. The present results are consistent with clinical observations $(5,6)$, and may suggest that frequent sneezing is the strongest symptom during the early-phase reaction and LPR of AR.

There is universal agreement that histamine is the primary mediator involved in the development of sneezing (29-31). During the course of AR, inflammatory cells release histamine. The histamine stimulates $\mathrm{H} 1$ receptors of sensory nerve endings in the nasal mucosa (32). By a nerve reflex, multiple sneezing is caused exclusively by histamine. In allergic inflammation, histamine is released by mast cells and basophils. It is well established that mast cells releasing histamine are the major cytokine source inducing sneezing in the early-phase reaction (29). A study by Schleimer et al (33) investigated the inflammatory cytokines in the LPR in humans. In the LPR, histamine and N- $\alpha$-tosyl-Larginine methyl esterase were found in the nasal secretions of patients exhibiting an LPR following antigen provocation tests. By contrast, prostaglandin D2 (PGD2) was not found. Since basophils do not generate PGD2, histamine may be released by basophils in the LPR. Basophils releasing histamine may be the main inducer of frequent sneezing in the LPR. At the same time, the histamine contributes to the late phase of AR by exhibiting proinflammatory effects (34-37). Yamasaki et al (26) reported that histamine levels had two peaks in nasal lavage fluid in a guinea pig model of AR, at 20 min and $5 \mathrm{~h}$ after challenge, which coincided with the peak time of frequent sneezing in the present study. In a previous study, Wagenmann et al (6) demonstrated that histamine levels in the nasal secretions of patients with AR were significantly increased in the early and late phases subsequent to nasal allergen provocation; however, the changes in histamine levels in the nasal lavage fluid following challenge were not detected in the present study. It is unknown whether histamine also exhibits a double peak in the guinea pig model of AR; however, it was shown in the present study that terfenadine, as an antihistamine drug, inhibited the development of the biphasic sneezing in this model. This finding strongly suggests that histamine contributes to the development of the early and late phases of sneezing.

In the present study, it was found that the guinea pigs in the model group produced significantly more sneezing in the first peak between the 19th and 30th challenges than between the first and 18th challenges $(\mathrm{P}<0.01)$. These results suggest that biphasic responders showed significantly severer symptoms during the early-phase reaction than single-phase responders, which is similar to observations in human $\operatorname{AR}(3,38)$. The mechanism of the above clinical phenomenon remains unclear. In a previous study, Imai et al (39) demonstrated that chronic eosinophil accumulation was induced by repeated antigen challenges in the nasal tissue at the early phase. The eosinophils may have been responsible for the amplification of the early-phase reaction, such as vascular permeability and mucosal edema (39). Milanese et al (40) subsequently reported that the eosinophil number was higher during the early phase in AR with LPR than that in AR with only the early-phase reaction. From these studies, it can be inferred that eosinophils may have an important role in the early-phase reaction of allergic inflammation; however, its participation in LPR has also been reported by numerous studies $(41,42)$. 
In conclusion, this study has confirmed that SEB can be used as an allergen to induce experimental AR with biphasic sneezing by repeated intranasal instillation. The SEB-sensitized guinea pigs are characterized by eosinophil infiltration in the nasal mucosa and detectable levels of allergen-specific IgE and $\operatorname{IgG}_{1}$ in the serum. The above indicators verify the successful establishment of the AR animal model with biphasic sneezing. Histamine may play an important role in the early- and late-phase sneezing in the model of AR. This model closely reflects AR and is a useful tool to study the association between SEB, AR and the pathogenesis of the late phase of AR. Furthermore, this model can be potentially used for the investigation of new drugs.

\section{Acknowledgements}

This study was supported by the National Natural Science Foundation Project (no. 81271061) and the National Key Clinical Specialties Construction Program of China (no. 81271061).

\section{References}

1. Meltzer EO: The pharmacological basis for the treatment of perennial allergic rhinitis and non-allergic rhinitis with topical corticosteroids. Allergy 52 (Suppl): 33-40, 1997.

2. Niedoszytko M, Chełmińska M, Chełmiński K, et al: Late-phase allergic reaction in nasal provocation with fungal allergens. Allergy Asthma Proc 29: 35-39, 2008.

3. de Graaf-in't Veld C, Garrelds IM, van Toorenenbergen AW and Gerth van Wijk R: Nasal responsiveness to allergen and histamine in patients with perennial rhinitis with and without a late phase response. Thorax 52: 143-148, 1997.

4. Wang D and Clement P: Assessment of early- and late-phase nasal obstruction in atopic patients after nasal allergen challenge. Clin Otolaryngol Allied Sci 20: 368-373, 1995.

5. Jordan TR, Rasp G, Pfrogner E and Kramer MF: An approach of immunoneurological aspects in nasal allergic late phase. Allergy Asthma Proc 26: 382-390, 2005.

6. Wagenmann M, Schumacher L and Bachert C: The time course of the bilateral release of cytokines and mediators after unilateral nasal allergen challenge. Allergy 60: 1132-1138, 2005.

7. Oldenbeuving NB, KleinJan A, Mulder PG, et al: Evaluation of an intranasal house dust mite provocation model as a tool in clinical research. Allergy 60: 751-759, 2005.

8. Bousquet J, Chanez P and Michel FB: Pathophysiology and treatment of seasonal allergic rhinitis. Respir Med 84 (Suppl A): 11-17, 1990.

9. Naclerio RM: Pathophysiology of perennial allergic rhinitis. Allergy 52 (Suppl): 7-13, 1997.

10. Nabe T, Mizutani N, Shimizu K, et al: Development of pollen-induced allergic rhinitis with early and late phase nasal blockage in guinea pigs. Inflamm Res 47: 369-374, 1998.

11. Zhao Y, van Hasselt CA, Woo KS et al: Establishment of a modified intranasally ovalbumin induced animal model of allergic rhinitis. Zhonghua Er Bi Yan Hou Tou Jing Wai Ke Za Zhi 40: 176-180, 2005 (In Chinese).

12. Tang X, Sun R, Hong S, et al: Repeated intranasal instillation with staphylococcal enterotoxin B induces nasal allergic inflammation in guinea pigs. Am J Rhinol Allergy 25: 176-181, 2011.

13. Breuer K, Haussler S, Kapp A and Werfel T: Staphylococcus aureus: colonizing features and influence of an antibacterial treatment in adults with atopic dermatitis. Br J Dermatol 147: 55-61, 2002.

14. Chambers HF: The changing epidemiology of Staphylococcusaureus? Emerg Infect Dis 7: 178-182, 2001.

15. Becker K, Friedrich AW, Lubritz G, et al: Prevalence of genes encoding pyrogenic toxin superantigens and exfoliative toxins among strains of Staphylococcus aureus isolated from blood and nasal specimens. J Clin Microbiol 41: 1434-1439, 2003.

16. Sakairi T, Suzuki K, Makita S, et al: Effects of fexofenadine hydrochloride in a guinea pig model of antigen-induced rhinitis Pharmacology 75: 76-86, 2005.

17. Avincsal MO, Ozbal S, Ikiz AO, Pekcetin C and Güneri EA: Effects of topical intranasal doxycycline treatment in the rat allergic rhinitis model. Clin Exp Otorhinolaryngol 7: 106-111, 2014.
18. Underwood DC, Osborn RR and Hand JM: Lack of late-phase airway responses in conscious guinea pigs after a variety of antigen challenges. Agents Actions 37: 191-194, 1992.

19. Zhao XJ: Experimental models of nasal hypersensitive reaction. Zhonghua Er Bi Yan Hou Ke Za Zhi 28: 17-8, 58-9, 1993 (In Chinese).

20. Okano M, Nishizaki K, Abe M, et al: Strain-dependent induction of allergic rhinitis without adjuvant in mice. Allergy 54: 593-601, 1999.

21. van de Rijn M, Mehlhop PD, Judkins A, et al: A murine model of allergic rhinitis: studies on the role of IgE in pathogenesis and analysis of the eosinophil influx elicited by allergen and eotaxin. J Allergy Clin Immunol 102: 65-74, 1998.

22. Wang LF, Xu LJ, Guo FH, et al: Effect of antiallergic herbal agents on chloride channel-3 and immune microenvironment in nasal mucosal epithelia of allergic rhinitis rabbits. Chin Med J (Engl) 123: 1034-1038, 2010.

23. Tsunematsu M, Yamaji T, Kozutsumi D, et al: A new murine model of allergic rhinitis by repeated intranasal Cry $\mathrm{j} 1$ challenge. Biomed Res 29: 119-123, 2008.

24. Zhao Y, Woo JK, Leung PC, et al: Symptomatic and pathophysiological observations in a modified animal model of allergic rhinitis. Rhinology 43: 47-54, 2005.

25. Lei F, Zhu D, Sun J and Dong Z: Effects of minimal persistent inflammation on nasal mucosa of experimental allergic rhinitis. Am J Rhinol Allergy 24: e23-e28, 2010.

26. Yamasaki M, Mizutani N, Sasaki K, et al: Involvement of thromboxane A2 and peptide leukotrienes in early and late phase nasal blockage in a guinea pig model of allergic rhinitis. Inflamm Res 50: 466-473, 2001.

27. Brozmanova M, Bartos V, Plank L and Tatar M: Experimental allergic rhinitis-related cough and airway eosinophilia in sensitized guinea pigs. J Physiol Pharmacol 58 (Suppl 5): 57-65, 2007.

28. Nabe T, Kubota K, Mizutani N, et al: Effect of local nasal immunotherapy on nasal blockage in pollen-induced allergic rhinitis of Guinea pigs. Allergol Int 57: 419-427, 2008.

29. Grønborg H, Bisgaard H, Rømeling F and Mygind N: Early and late nasal symptom response to allergen challenge. The effect of pretreatment with a glucocorticosteroid spray. Allergy 48: 87-93, 1993.

30. Doyle WJ, Boehm S and Skoner DP: Physiologic responses to intranasal dose-response challenges with histamine, methacholine, bradykinin, and prostaglandin in adult volunteers with and without nasal allergy. J Allergy Clin Immunol 86: 924-935, 1990.

31. Svensson C, Baumgarten CR, Pipkorn U, et al: Reversibility and reproducibility of histamine induced plasma leakage in nasal airways. Thorax 44: 13-18, 1989.

32. White MV and Kaliner MA: Mediators of allergic rhinitis. J Allergy Clin Immunol 90: 699-704, 1992.

33. Schleimer RP, Fox CC, Naclerio RM, et al: Role of human basophils and mast cells in the pathogenesis of allergic diseases. J Allergy Clin Immunol 76: 369-374, 1985.

34. Horak F: Clinical advantages of dual activity in allergic rhinitis. Allergy 55 (Suppl 64): 34-39, 2000.

35. Montoro J, Sastre J, Jáuregui I, et al: Allergic rhinitis: continuous or on demand antihistamine therapy? J Investig Allergol Clin Immunol 17 Suppl 2: 21-27, 2007.

36. Pietrzkowicz M and Grzelewska-Rzymowska I: Histamine as a mediator of allergic inflammation. Pol Merkur Lekarski 6: 232-235, 1999 (In Polish).

37. Pawankar R, Yamagishi S and Yagi T: Revisiting the roles of mast cells in allergic rhinitis and its relation to local IgE synthesis. Am J Rhinol 14: 309-317, 2000.

38. Terada N, Hamano N, Hohki G, et al: Late phase response in nasal mucosa closely correlated with immediate phase reaction and hyperreactivity to histamine. Acta Otolaryngol 118: 392-397, 1998.

39. Imai N, Miyahara A, Yamazaki Y, et al: Involvement of eosinophils in the early-phase allergic reaction in a guinea pig rhinitis model. Int Arch Allergy Immunol 122: 270-278, 2000.

40. Milanese M, Ricca V, Canonica GW and Ciprandi G: Eosinophils, specific hyperreactivity and occurrence of late phase reaction in allergic rhinitis. Eur Ann Allergy Clin Immunol 37: 7-10, 2005.

41. Elovsson S, Smailagic A, Erjefalt I, et al: Evaluation of nasal barrier dysfunction at acute- and late-phase reactions in a guinea pig model of allergic rhinitis. Vascul Pharmacol 43: 267-276, 2005.

42. Sedgwick JB, Calhoun WJ, Gleich GJ, et al: Immediate and late airway response of allergic rhinitis patients to segmental antigen challenge. Characterization of eosinophil and mast cell mediators. Am Rev Respir Dis 144: 1274-1281, 1991. 